\title{
Optimal Treatment Strategies for HIV with Antibody Response
}

\author{
Yinggao Zhou, ${ }^{1}$ Kuan Yang, ${ }^{2}$ Kai Zhou, ${ }^{1}$ and Chunling Wang ${ }^{1}$ \\ ${ }^{1}$ School of Mathematics and Statistics, Central South University, Changsha, Hunan 410083, China \\ ${ }^{2}$ School of Business Adminstration, Hunan University, Changsha, Hunan 410082, China \\ Correspondence should be addressed to Kuan Yang; yangkuan@hnu.edu.cn
}

Received 21 April 2014; Revised 6 June 2014; Accepted 24 June 2014; Published 10 July 2014

Academic Editor: Zhen Jin

Copyright (C) 2014 Yinggao Zhou et al. This is an open access article distributed under the Creative Commons Attribution License, which permits unrestricted use, distribution, and reproduction in any medium, provided the original work is properly cited.

\begin{abstract}
Numerical analysis and optimization tools are used to suggest improved therapies to try and cure HIV infection. An HIV model of ordinary differential equation, which includes immune response, neutralizing antibodies, and multidrug effects, is improved. For a fixed time, single-drug and two-drug treatment strategies are explored based on Pontryagin's maximum principle. Using different combinations of weight factor pairs combining with special upper-bound pairs for controls, nine types of treatment policies are determined and different therapy effects are numerically simulated with a gradient projection method. Some strategies are effective, but some strategies are not particularly helpful for the therapy of HIV/AIDS. Comparing the effective treatment strategies, we find a more appropriate strategy with maximizing the number of uninfected $\mathrm{CD} 4^{+} \mathrm{T}$-cells and minimizing the number of active virus.
\end{abstract}

\section{Introduction}

Up to date, drug treatments are still available control methods of HIV/AIDS. Reverse transcriptase inhibitors (RTIs) can inhibit HIV RNA from being converted into DNA, thus blocking integration of the viral code into the target cell. Protease inhibitors (PIs) function by preventing the assembly of key viral proteins after they have been produced by the infected host cell. Therefore, PIs effectively reduce the number of infectious virus particles released by an infected cell. HAART consists of combined drug regimens that include two, or three, nucleoside agents alone or two nucleoside agents combined with a protease inhibitor, or a nonnucleoside reverse transcriptase inhibitor [1]. This paper explores the effects of a combination of a protease inhibitor and a nucleoside reverse transcriptase inhibitor.

Mathematical models are often used to study HIV/AIDS spread and host-drug-virus interactions to make assumptions and to suggest new methods for its optimal control. Zurakowski and Teel [2] developed a model to determine optimal treatment interruption schedules, simulate therapeutic vaccine, and induce Cytotoxic T Lymphocyte (CTL) mediated control of HIV infection. Karrakchou et al. [3] proposed an infectious model which described the interaction of HIV virus and the immune system of the human body to investigate the fundamental role of chemotherapy treatment in controlling the virus reproduction and to determine the optimal methodology for administering antiviral medication therapies to fight HIV infection. Garira et al. [4] studied an optimal control problem including immune response and multidrug effects for HIV multitherapy enhancement. However, although most of the previous researches took the Cytotoxic T Lymphocytes (CTL) into account, they ignored the neutralizing antibodies, and very few studies have been carried out on comparing the outcomes before and after using antiviral drugs.

In this paper, we use numerical analysis and optimization tools to suggest improved therapies to try and cure HIV infection for an improved HIV model of ordinary differential equation, which includes immune response, neutralizing antibodies, and multidrug effects. We use two controls, one simulating effect of RTIs, and the other control simulating effect of PIs. We determine nine types of treatment strategies, which including single-drug controls and two-drug controls, based on different combinations of weight factor pairs combining with special upper-bound pairs for controls. The rest of this paper is organized as follows. In Section 2, an HIV model with antibody response is extended and an optimal control problem for HIV/AIDS is established. In Section 3, the existence of optimal control pair is investigated. 
In Section 4, the optimal control pair (the optimality system) is derived by using Pontryagin's maximum principle [5-7]. In Section 5, some numerical results of optimal treatment policies are illustrated. In Section 6, we conclude by discussing the results of the numerical simulations based on different weight coefficients and upper-bounds for controls.

\section{The Model}

In 2005, an optimal control problem including immune response and multidrug effects for HIV multitherapy enhancement,

$$
\begin{aligned}
& \max \quad J= \int_{0}^{t_{f}}\left[T-\left(\alpha_{1} u_{1}^{2}+\alpha_{2} u_{2}^{2}\right)\right] d t \\
& \text { s.t. } \quad \dot{T}= s_{1}+\frac{r_{1} T V}{B_{V}+V}-\mu_{T} T-\left(1-u_{1}\right) k_{1} T V e^{-a_{0} E} \\
&-\frac{r_{2} T V}{B_{T}+T}, \\
& \dot{I}=\left(1-u_{1}\right) k_{1} T V e^{-a_{0} E}-\mu_{I} I-k_{3} I E \\
& \dot{V}=\left(1-u_{2}\right) N \mu_{I} I e^{-a_{1} E}-\mu_{V} V, \\
& \dot{E}= s_{2}+k_{4} T V E-\mu_{E} E, \\
& T(0), I(0), V(0), E(0) \geq 0, \quad 0 \leq u_{1}, u_{2} \leq 1
\end{aligned}
$$

was studied by Garira et al. [4]. For a fixed time, a twodrug treatment strategy was obtained based on Pontryagin's minimum principle. The quadratic objective function considered takes into account two contributions: the viral load and the quantities of drug used. Simulations were carried out using an indirect optimization method. At each step the differential system was solved using Runge-Kutta five order scheme. Results highlighted that a progressive reduction of reverse transcriptase inhibitor (RTIs) drug dose on the one hand along with on the other hand a progressive increase of protease inhibitor (PIs) one was needed for optimality.

Garira et al. [4] take the cytotoxic T lymphocytes (CTL) into account, however, they ignore the neutralizing antibodies and latent period. The antibodies can combine with the virus such that the virus cannot get into target cells and protect a host against the infection by HIV-1. They can be induced several weeks after infection [8-11]. These facts mean that the neutralizing antibodies are important in the early stage of the infection. Because the antibodies are secreted by effector B cells, we add a term $B(t)$, which represents the concentration of effector B cells, to the control system. Since the differentiation and proliferation of B-cells to effector Bcells need the help of CD $4^{+}$T-cells, we assume the generation rate is $k_{5} V T$. A simple mass-action type of term will be used with rate constant $k_{5}$. As HIV-1 mutates rapidly, the average term of validity of effector B-cells is shorter than normal, we multiply the death rate, $\mu_{B}$, by a positive constant $\beta \geq 1$. Thus, the term $B(t)$ should satisfy the following equation:

$$
\dot{B}=k_{5} V T-\beta \mu_{B} B .
$$

Because of the assumption that the antibodies' concentration is proportional to effector B cells' concentration, the neutralizing rate should be expressed by $q B V$ and (4) should be modified to the following equation:

$$
\dot{V}=\left(1-u_{2}\right) N \mu_{I} I e^{-a_{1} E}-\mu_{V} V-q B V .
$$

Since viral DNA once integrated in cell nucleus, may remain dormant, in the latent stage, we also take the latent period into account as in [11, 12]. Furthermore, we assume that latently infected cells, which have not yet produced virus, switch to productively infected cell with rate $k_{2}$. Let $L$ be the concentration of latently infected $\mathrm{CD} 4{ }^{+} \mathrm{T}$ cells, then we have

$$
\dot{L}=\omega\left(1-u_{1}\right) k_{1} T V e^{-a_{0} E}-\mu_{T} L-k_{2} L,
$$

which implies that (3) should be modified to the following equation:

$$
\dot{I}=(1-\omega)\left(1-u_{1}\right) k_{1} T V e^{-a_{0} E}+k_{2} L-\mu_{I} I-k_{3} I E,
$$

where $\omega$ represents fraction of latently infected $\mathrm{CD} 4^{+} \mathrm{T}$ cells in infected cells production, and so the term $\omega(1-$ $\left.u_{1}\right) k_{1} T V e^{-a_{0} E}$ is added to $L$ and $(1-\omega)\left(1-u_{1}\right) k_{1} T V e^{-a_{0} E}$ is added to $I$.

Thus, a new HIV treatment system is established as the following system:

$$
\begin{gathered}
\dot{T}=s_{1}+\frac{r_{1} T V}{B_{V}+V}-\mu_{T} T-\left(1-u_{1}\right) k_{1} T V e^{-a_{0} E} \\
-\frac{r_{2} T V}{B_{T}+T}, \\
\dot{L}=\omega\left(1-u_{1}\right) k_{1} T V e^{-a_{0} E}-\mu_{T} L-k_{2} L, \\
\dot{I}=(1-\omega)\left(1-u_{1}\right) k_{1} T V e^{-a_{0} E}+k_{2} L-\mu_{I} I-k_{3} I E, \\
\dot{V}=\left(1-u_{2}\right) N \mu_{I} I e^{-a_{1} E}-\mu_{V} V-q B V, \\
\dot{E}=s_{2}+k_{4} T V E-\mu_{E} E, \\
\dot{B}=k_{5} T V-\beta \mu_{B} B, \\
T(0), I(0), V(0), E(0) \geq 0, \quad 0 \leq u_{1} \leq b_{1}, \\
0 \leq u_{2} \leq b_{2}, \quad 0 \leq b_{1}, b_{2} \leq 1,
\end{gathered}
$$

where $T, L, I, V, E, B$ denote the concentration of uninfected $\mathrm{CD} 4^{+} \mathrm{T}$ cells, latently infected $\mathrm{CD} 4^{+} \mathrm{T}$ cells, actively infected cells, $\mathrm{CD}^{+} \mathrm{T}$ cells, infectious viruses, cytotoxic lymphocytes effector, and $\mathrm{B}$ cells, respectively. Drugs efficiency is represented by the controls $u_{1}$ and $u_{2}$ which account for reverse transcriptase inhibitors and protease inhibitors actions, respectively.

This is a modification of model for HIV infection considered in Garira et al. [4]. In view of the importance of neutralizing antibody especially in the early stage of the infection, we add a term $B(t)$ as in Zhou et al. [11], which describes effector $B$ cells, to the control system. We chose effector B cells as antibody response because the antibodies are secreted by effector B cells. 
TABLE 1: Definitions and values for parameters.

\begin{tabular}{|c|c|c|}
\hline Parameter & Constants & Values with unit \\
\hline$r_{1}$ & Rate growth of uninfected $\mathrm{CD} 4^{+} \mathrm{T}$ & $0.01 \mathrm{~d}^{-1}$ \\
\hline$r_{2}$ & Rate apoptosis of uninfected $\mathrm{CD} 4^{+} \mathrm{T}$ & $2 e-3 \mathrm{~d}^{-1}$ \\
\hline$B_{V}$ & Parameter involving amount of antigen & 400 \\
\hline$B_{T}$ & Parameter involving apoptosis of $\mathrm{CD} 4^{+} \mathrm{T}$ & 350 \\
\hline$\mu_{T}$ & Death rate of uninfected $\mathrm{CD} 4^{+} \mathrm{T}$ & $0.02 \mathrm{~d}^{-1}$ \\
\hline$\mu_{I}$ & Death rate of infected $\mathrm{CD} 4^{+} \mathrm{T}$ & $0.25 \mathrm{~d}^{-1}$ \\
\hline$\mu_{V}$ & Death rate of virus & $0.95 \mathrm{~d}^{-1}$ \\
\hline$\mu_{E}$ & Death rate of CTL & $1.5 \mathrm{~d}^{-1}$ \\
\hline$\mu_{B}$ & Death rate of antibodies & $0.005 \mathrm{~d}^{-1}$ \\
\hline$q$ & Rate virus deleted by CTL & $5 e-2 \mathrm{~mm}^{3} \mathrm{~d}^{-1}$ \\
\hline$k_{1}$ & Rate $\mathrm{CD} 4^{+} \mathrm{T}$ becomes infected by virus & $2 e-4 \mathrm{~mm}^{3} \mathrm{~d}^{-1}$ \\
\hline$k_{2}$ & Rate latently infected convert to actively infected & $3 e-3 \mathrm{~mm}^{3} \mathrm{~d}^{-1}$ \\
\hline$k_{3}$ & Rate actively infected cells deleted by CTL & $2 e-3 \mathrm{~mm}^{3} \mathrm{~d}^{-1}$ \\
\hline$k_{4}$ & Rate growth of CTL & $1 e-5 \mathrm{~mm}^{3} \mathrm{~d}^{-1}$ \\
\hline$k_{5}$ & Rate growth of antibody & $2 e-4 \mathrm{~mm}^{3} \mathrm{~d}^{-1}$ \\
\hline$a_{0}$ & Efficiency of each CTL & $0.01 \mathrm{~d}^{-1}$ \\
\hline$a_{1}$ & Rate CTL suppresses virus & $0.075 \mathrm{~d}^{-1}$ \\
\hline$N$ & Average of viral particles & 1000 \\
\hline$s_{1}$ & Source term for uninfected $\mathrm{CD} 4^{+} \mathrm{T}$ & $20 \mathrm{~mm}^{3} \mathrm{~d}^{-1}$ \\
\hline$s_{1}$ & Source term for CTL & $10 \mathrm{~mm}^{3} \mathrm{~d}^{-1}$ \\
\hline$\omega$ & Fraction of latently/infected $\mathrm{CD} 4^{+} \mathrm{T}$ & 0.5 \\
\hline$\beta$ & Multiple of death rate of antibodies & 10 \\
\hline
\end{tabular}

Uninfected cells are generated at a constant rate $s_{1}$ and are proliferated at the rate $r_{1} T V / B_{V}+V$, where $r_{1}$ is the proliferation rate and $B_{V}$ is a parameter that determines the amount of antigen needed to generate half maximal stimulation. Uninfected cells die at a rate of $\mu_{T} T$, implying that the average lifetime of an uninfected cell is $1 / \mu_{T}$, and destruct at a rate of $r_{2} T V / B_{T}+T$ due to the influence of toxic viral proteins, where $r_{2}$ is the rate of apoptosis and $B_{T}$ is a parameter that determines the scale at which engagement of apoptosis receptors begins to take effect. Uninfected cells may become infected cells at a rate of $\left(1-u_{1}\right) k_{1} T V e^{-a_{0} E}$, where $k_{1}$ is the rate of infection of $\mathrm{CD}^{+} \mathrm{T}$ cells by virus and $a_{0}$ is the efficiency of each CTL in reducing $\mathrm{CD} 4^{+} \mathrm{T}$ cells infection while $u_{1}$ represents efficiency of RTIs in blocking the recoding process. Infected cells die at a rate of $\mu_{I} I$, meaning that the average lifetime of an infected cell is $1 / \mu_{I}$, and are lysed by CTLs at a rate of $k_{2} I E$. Free infectious virus is replicated at a rate of $\left(1-u_{2}\right) N \mu_{I} I e^{a_{1} E}$, where an average of $N$ virus particles are released per infected cell and $a_{1}$ is the rate at which each CTL suppresses virus production while $u_{2}$ represents efficiency of PIs in reducing infection.

Using the method in [13] combined with the least square estimation, we fit the parameters $q, k_{5}, \beta, \mu_{B}$ keeping the rest of parameters in [4] unchanged; the fitting data comes from the literature [14]. We employ the data of patient 7 and patient 9 in [14] to estimate the parameters and get the range of the parameters $q: 0.0089-0.0549, k_{4}: 0.0038-0.000382$, $\beta \mu_{B}=0.4104-0.0506$.
Thus, definitions and values of the parameters used in this model are given in Table 1 (see also [4] with references for old parameters).

Our objective functional is also defined as

$$
J\left(u_{1}, u_{2}\right)=\int_{t_{0}}^{t_{f}}\left[T-\left(\alpha_{1} u_{1}^{2}+\alpha_{2} u_{2}^{2}\right)\right] d t .
$$

The first term represents the benefit of $\mathrm{T}$ cells and other terms are systemic costs of drug treatments. The positive constants $\alpha_{1}$ and $\alpha_{2}$ balance the size of the terms, and $u_{1}^{2}, u_{2}^{2}$ reflect the severity of the side effects of the drugs. We seek an optimal control pair, $u_{1}^{*}, u_{2}^{*}$, such that

$$
J\left(u_{1}^{*}, u_{2}^{*}\right)=\max _{0 \leq u_{1} \leq b_{1}, 0 \leq u_{2} \leq b_{2}} J\left(u_{1}, u_{2}\right) .
$$

\section{Existence of an Optimal Control Pair}

Theorem 1. For the control problem (11), there exists an optimal control pair $\left(u_{1}^{*}, u_{2}^{*}\right)$ that maximizes the objective functional $J\left(u_{1}, u_{2}\right)$.

Proof. To use an existence result, Theorem III.4.1 from [15], we must check the following properties.

(1) The set of controls and corresponding state variables is nonempty.

(2) The control $U$ set is convex and closed. 

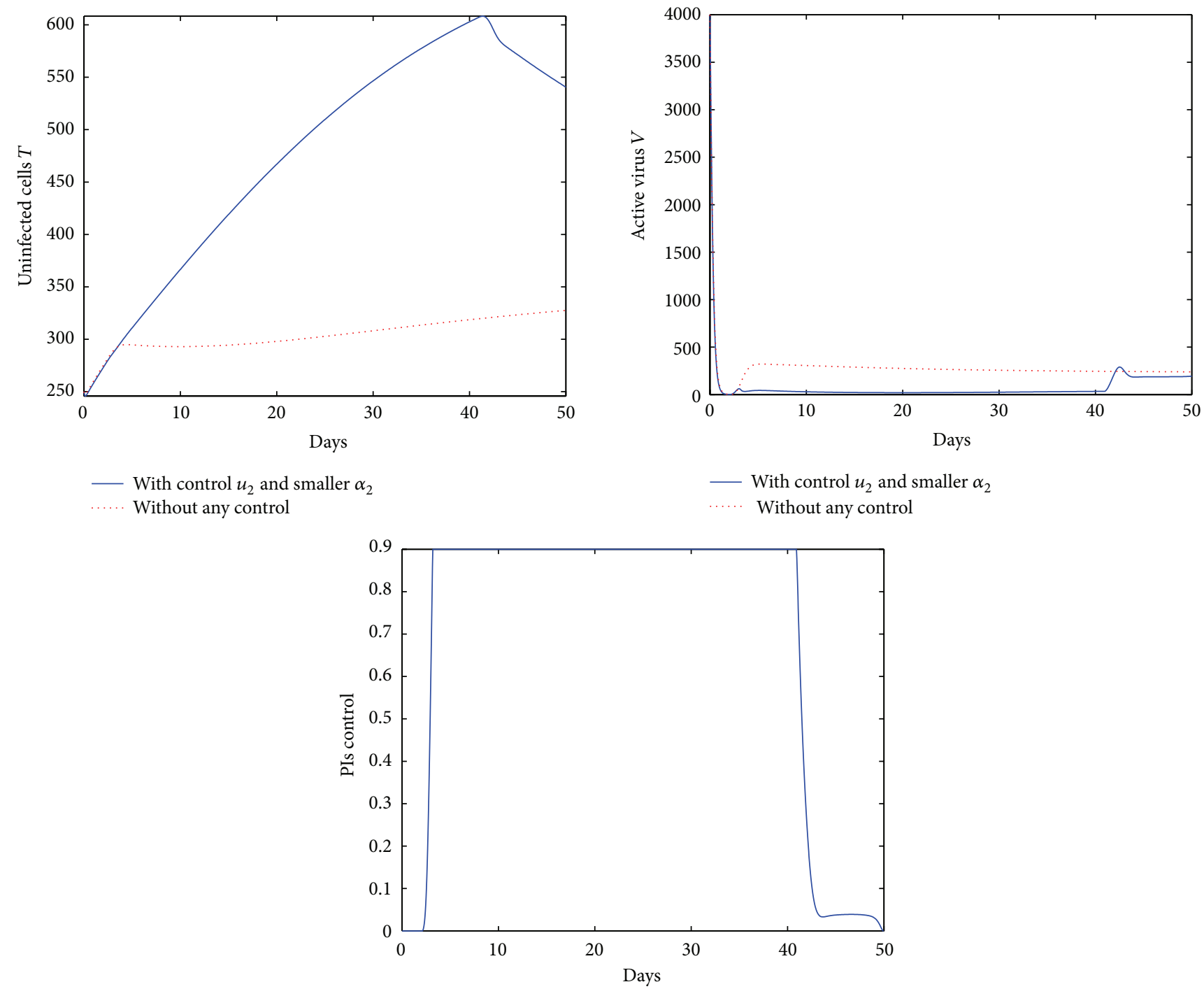

FIgURE 1: Optimal solutions only using PIs with sufficiently small weight coefficient $\alpha_{2}$ for a 50-day treatment.

(3) The right hand side of the state system is bounded by a linear function in the state and control variables.

(4) The integrand of the objective functional is concave on $U$.

(5) There exist constants $c_{1}, c_{2}>0$ and $b>1$ such that the integrand of the objective functional is bounded above by

$$
c_{2}-c_{1}\left(\left|u_{1}\right|^{2}+\left|u_{2}\right|^{2}\right)^{b / 2}
$$

First, an existence result in Lukes ([16], Theorem 9.2.1) for the control system (11) for bounded coefficients is invoked, which gives condition 1 . The control set $U=\left\{\left(u_{1}, u_{2}\right): 0 \leq\right.$ $\left.u_{1} \leq b_{1}, 0 \leq u_{2} \leq b_{2}\right\}$ is closed and convex by definition. Since the control system is bilinear in $u_{1}, u_{2}$, the right hand side of (11) satisfies condition 3 , using the boundedness of the solutions obtained by the analytical method in [17]. Note that the integrand of the objective functional is concave on the admissible control set $U$. Also we have the last condition needed

$$
T-\left(\alpha_{1} u_{1}^{2}+\alpha_{2} u_{2}^{2}\right) \leq c_{2}-c_{1}\left(\left|u_{1}\right|^{2}+\left|u_{2}\right|^{2}\right)^{b / 2}
$$

where $c_{2}$ depends on the upper-bound on $T$ and $c_{1}>0$ since $\alpha_{1}, \alpha_{2}>0$. We conclude that there exists an optimal control pair. This completes the proof.

\section{Optimality System}

Denote Hamiltonian $H\left(T, L, I, V, E, B ; u_{1}, u_{2} ; \lambda_{1}, \lambda_{2}, \lambda_{3}, \lambda_{4}\right.$, $\left.\lambda_{5}, \lambda_{6}\right)$ as

$$
\begin{aligned}
H= & T-\left(\alpha_{1} u_{1}^{2}+\alpha_{2} u_{2}^{2}\right) \\
& +\lambda_{1}\left(s_{1}+\frac{r_{1} T V}{B_{V}+V}-\mu_{T} T-\left(1-u_{1}\right) k_{1} T V e^{-a_{0} E}\right.
\end{aligned}
$$



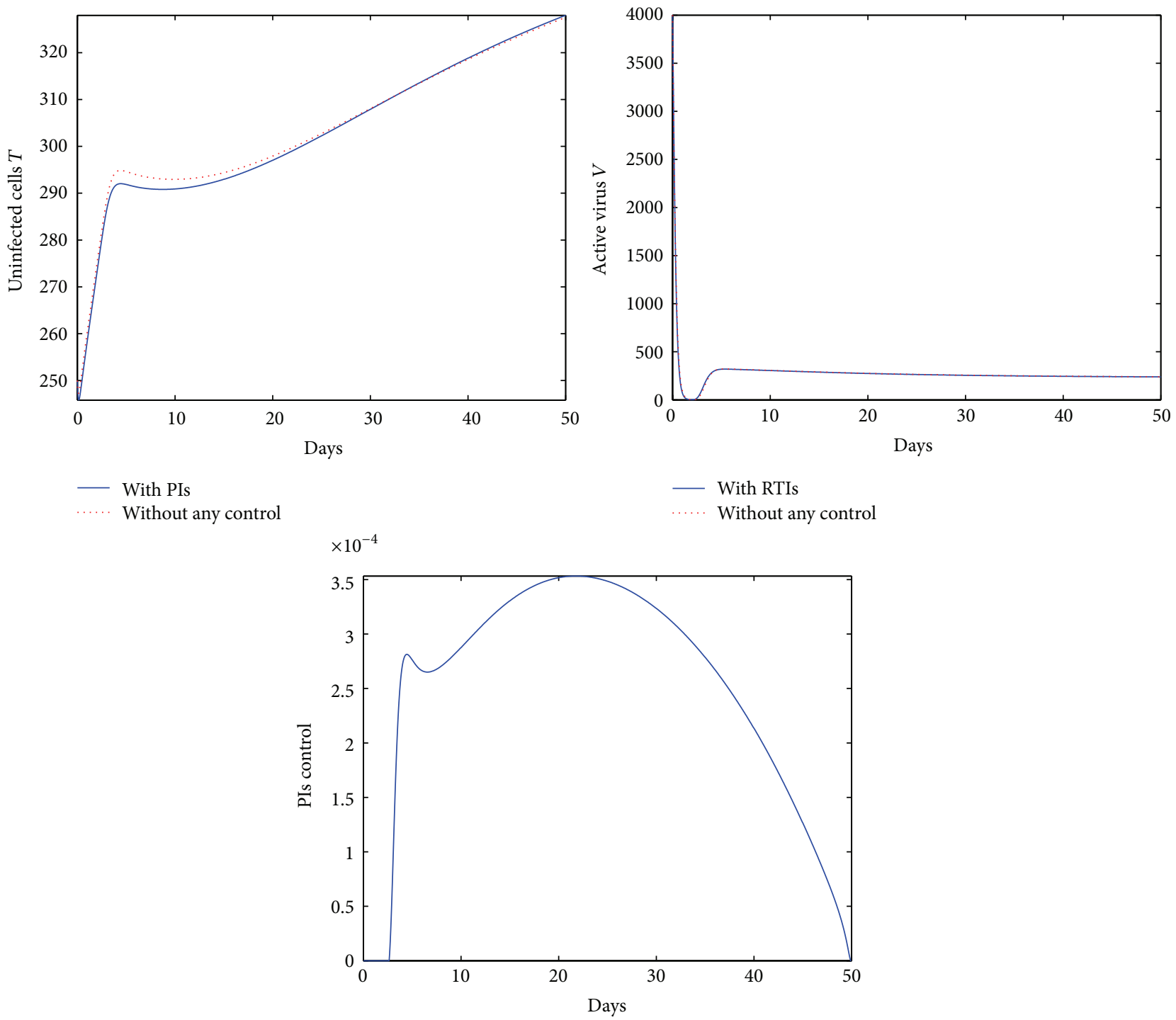

FIGURE 2: Optimal solutions only using PIs with sufficiently large weight coefficient $\alpha_{2}$ for a 50-day treatment.

$$
\begin{aligned}
& \left.-\frac{r_{2} T V}{B_{T}+T}\right) \\
+ & \lambda_{2}\left(\omega\left(1-u_{1}\right) k_{1} T V e^{-a_{0} E}-\mu_{T} L-k_{2} L\right) \\
+ & \lambda_{3}\left((1-\omega)\left(1-u_{1}\right) k_{1} T V e^{-a_{0} E}+k_{2} L-\mu_{I} I-k_{3} I E\right) \\
+ & \lambda_{4}\left(\left(1-u_{2}\right) N \mu_{I} I e^{-a_{1} E}-\mu_{V} V-q B V\right) \\
+ & \lambda_{5}\left(s_{2}+k_{4} T V E-\mu_{E} E\right) \\
+ & \lambda_{6}\left(k_{5} T V-\beta \mu_{B} B\right),
\end{aligned}
$$

where $\lambda_{i}(i=1,2, \ldots, 6)$ are costate variables. By Pontryagin's maximum principle, we have the following Theorem.
Theorem 2. If $u_{1}^{*}, u_{2}^{*}$ are optimal controls of the optimal control problem (11)-(13), $T^{*}, L^{*}, I^{*}, V^{*}, E^{*}, B^{*}$ are the corresponding optimal paths, then there exist costate variables $\lambda_{i}$ $(i=1,2, \ldots, 6)$ such that, besides the fact that control system (11) is satisfied, the following conditions are satisfied:

(i) costate equations:

$$
\begin{array}{r}
\dot{\lambda}_{1}=-\frac{\partial H}{\partial T} \\
=-1+\lambda_{1}\left[\mu_{T}+\left(1-u_{1}\right) k_{1} V e^{-a_{0} E}\right. \\
\\
\left.\quad-\frac{r_{1} V}{B_{V}+V}+\frac{r_{2} B_{T} V}{\left(B_{T}+T\right)^{2}}\right]
\end{array}
$$




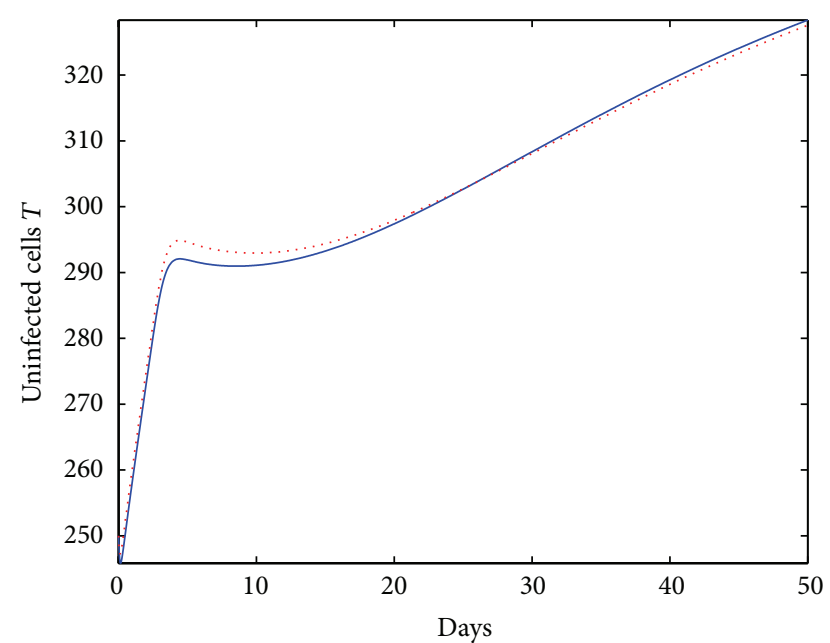

With control $u_{1}$ and larger $\alpha_{1}$

Without any control

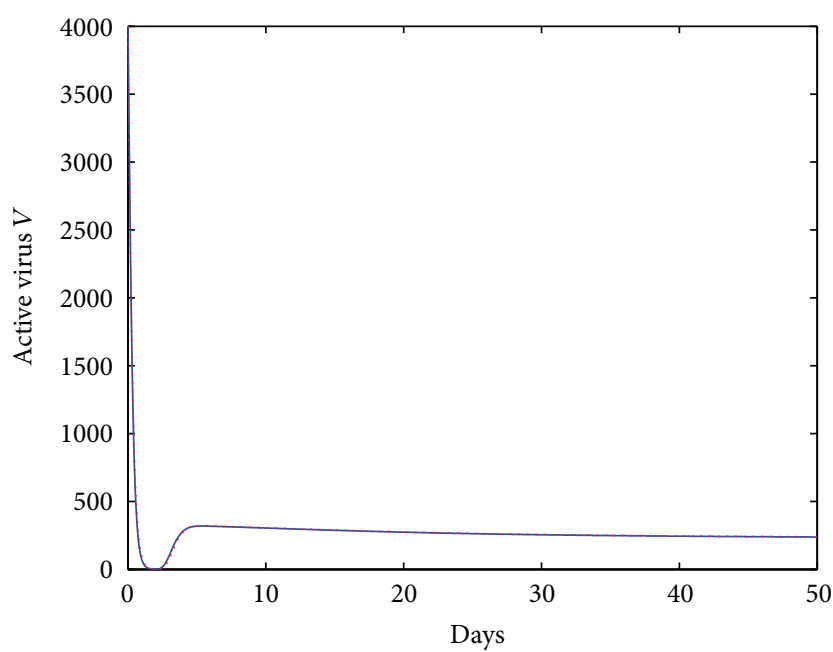

With control $u_{1}$ and larger $\alpha_{1}$

Without any control

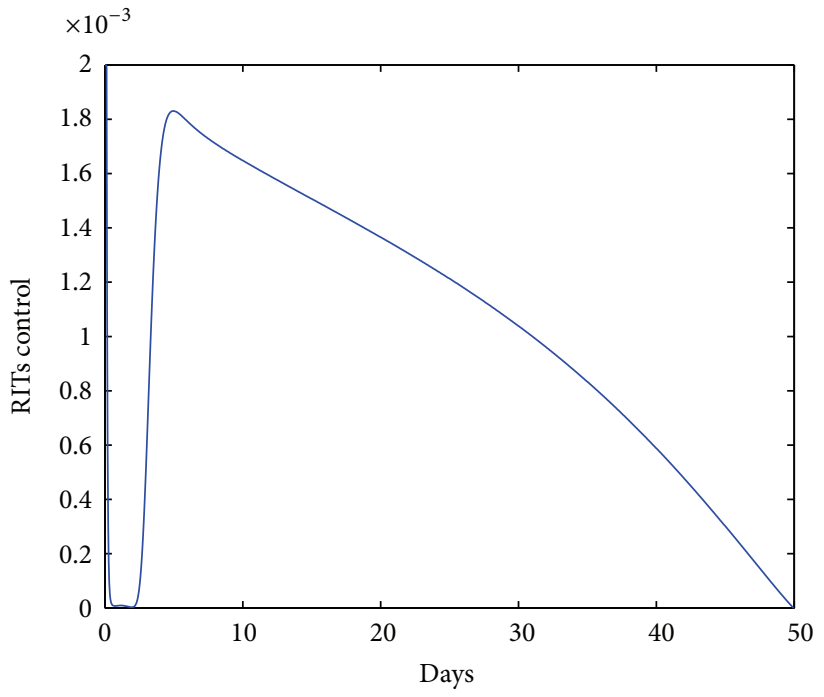

FIGURE 3: Optimal solutions only using RTIs with sufficiently large weight coefficient $\alpha_{1}$ for a 50-day treatment.

$$
\begin{gathered}
-\left(\omega \lambda_{2}+(1-\omega) \lambda_{3}\right)\left(1-u_{1}\right) k_{1} V e^{-a_{0} E} \\
-\lambda_{5} k_{4} V E-\lambda_{6} k_{5} V, \\
\dot{\lambda}_{2}=-\frac{\partial H}{\partial L}=\left(\mu_{T}+k_{2}\right) \lambda_{2}-k_{2} \lambda_{3}, \\
\dot{\lambda}_{3}=-\frac{\partial H}{\partial I} \\
=\lambda_{3}\left(\mu_{I}+k_{3} E\right)-\lambda_{4}\left(1-u_{2}\right) N \mu_{I} e^{-a_{1} E}, \\
\dot{\lambda}_{4}=-\frac{\partial H}{\partial V} \\
=\lambda_{1}\left[\left(1-u_{1}\right) k_{1} T e^{-a_{0} E}+\frac{r_{2} T}{B_{T}+T}-\frac{r_{1} B_{V} T}{\left(B_{V}+V\right)^{2}}\right] \\
-\left(\omega \lambda_{2}+(1-\omega) \lambda_{3}\right)\left(1-u_{1}\right) k_{1} T e^{-a_{0} E}
\end{gathered}
$$

$$
\begin{gathered}
+\lambda_{4}\left(\mu_{V}+q B\right)-\lambda_{5} k_{4} T E-\lambda_{6} k_{5} T, \\
\dot{\lambda}_{5}=-\frac{\partial H}{\partial E}=\left(\omega \lambda_{2}-\lambda_{1}\right) a_{0}\left(1-u_{1}\right) k_{1} T V e^{-a_{0} E} \\
+\lambda_{3}\left(a_{0}(1-\omega)\left(1-u_{1}\right) k_{1} T V e^{-a_{0} E}+k_{3} I\right) \\
+\lambda_{4} a_{1}\left(1-u_{2}\right) N \mu_{I} I e^{-a_{1} E}+\lambda_{5}\left(\mu_{E}-k_{4} T V\right), \\
\dot{\lambda}_{6}=-\frac{\partial H}{\partial B}=\lambda_{4} q V+\lambda_{6} \beta \mu_{B} ;
\end{gathered}
$$

(ii) optimality conditions:

$$
\begin{gathered}
H\left(T^{*}, L^{*}, I^{*}, V^{*}, E^{*}, B^{*} ; u_{1}^{*}, u_{2}^{*} ; \lambda_{1}, \lambda_{2}, \lambda_{3}, \lambda_{4}, \lambda_{5}, \lambda_{6}\right) \\
=\max _{0 \leq u_{i} \leq b_{i}, i=1,2} H\left(T^{*}, L^{*}, I^{*}, V^{*}, E^{*}, B^{*} ; u_{1}, u_{2} ;\right. \\
\left.\lambda_{1}, \lambda_{2}, \lambda_{3}, \lambda_{4}, \lambda_{5}, \lambda_{6}\right),
\end{gathered}
$$



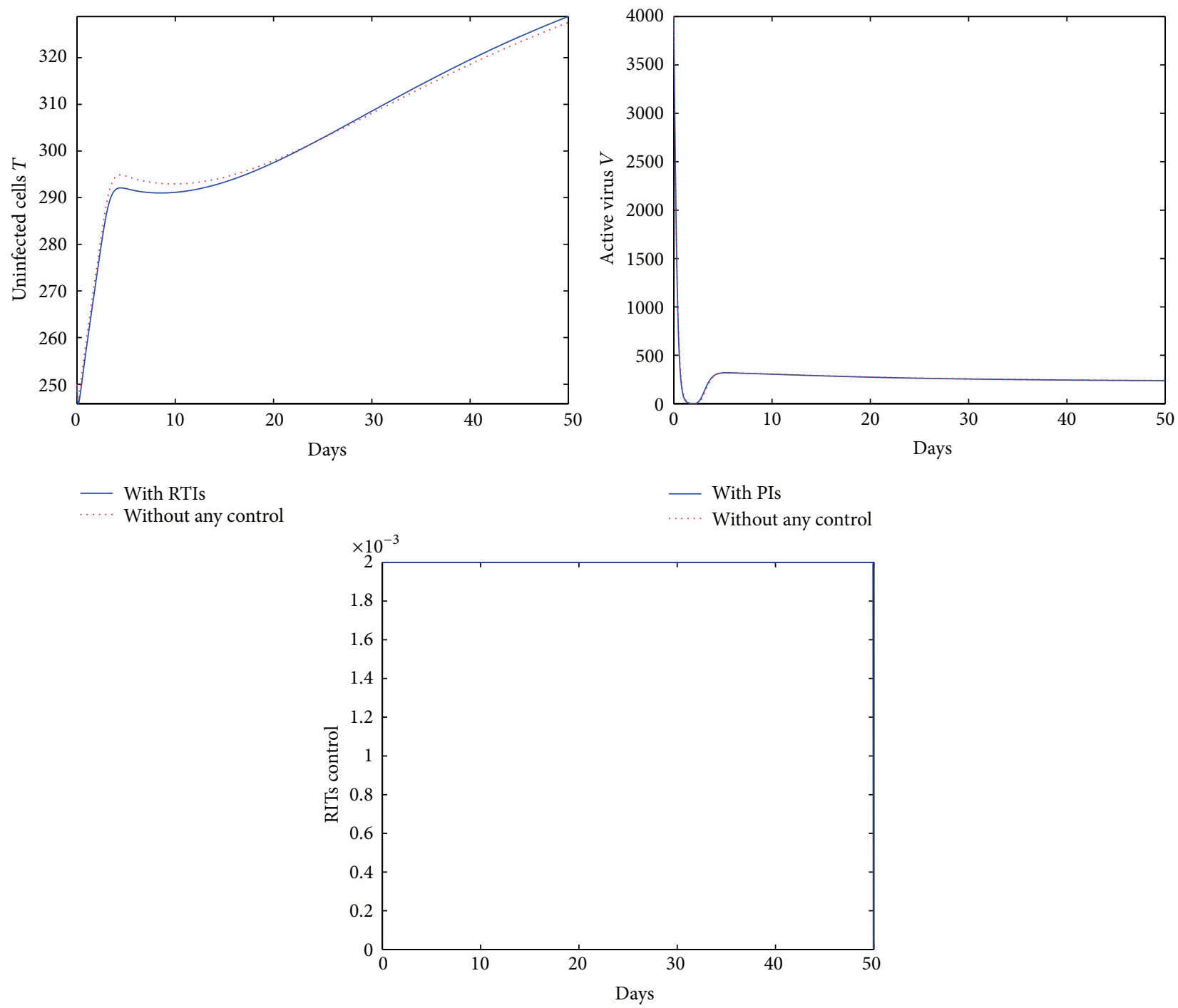

FIGURE 4: Optimal solutions only using RTIs with sufficiently small weight coefficient $\alpha_{1}$ for a 50-day treatment.

which imply that

$u_{1}^{*}$

$$
=\min \left\{b_{1}\right.
$$

$$
\max \left\{\frac{\left(\lambda_{1}-\omega \lambda_{2}-(1-\omega) \lambda_{3}\right) k_{1} T^{*} V^{*} e^{-a_{0} E^{*}}}{2 \alpha_{1}},\right.
$$$$
0\}\}
$$$$
u_{2}^{*}=\min \left\{b_{2}, \max \left\{-\frac{\lambda_{4} N \mu_{I} I^{*} e^{-a_{1} E^{*}}}{2 \alpha_{2}}, 0\right\}\right\} \text {; }
$$

(iii) transversality conditions:

$$
\lambda_{i}\left(t_{f}\right)=0, \quad i=1,2, \ldots, 6 .
$$

The optimality system consists of control system (11) coupled with the costate equation (17) with the initial conditions and transversality conditions together with the characterization of the optimal control pair (19).

Remark 3. Using similar method in [11], we can obtain the uniqueness of solution to the above optimality system when $t_{f}$ is sufficiently small. We omit it

\section{Numerical Illustration}

Analytical solutions for optimal control are difficult to obtain since the system is nonlinear. In this section, we use gradient 

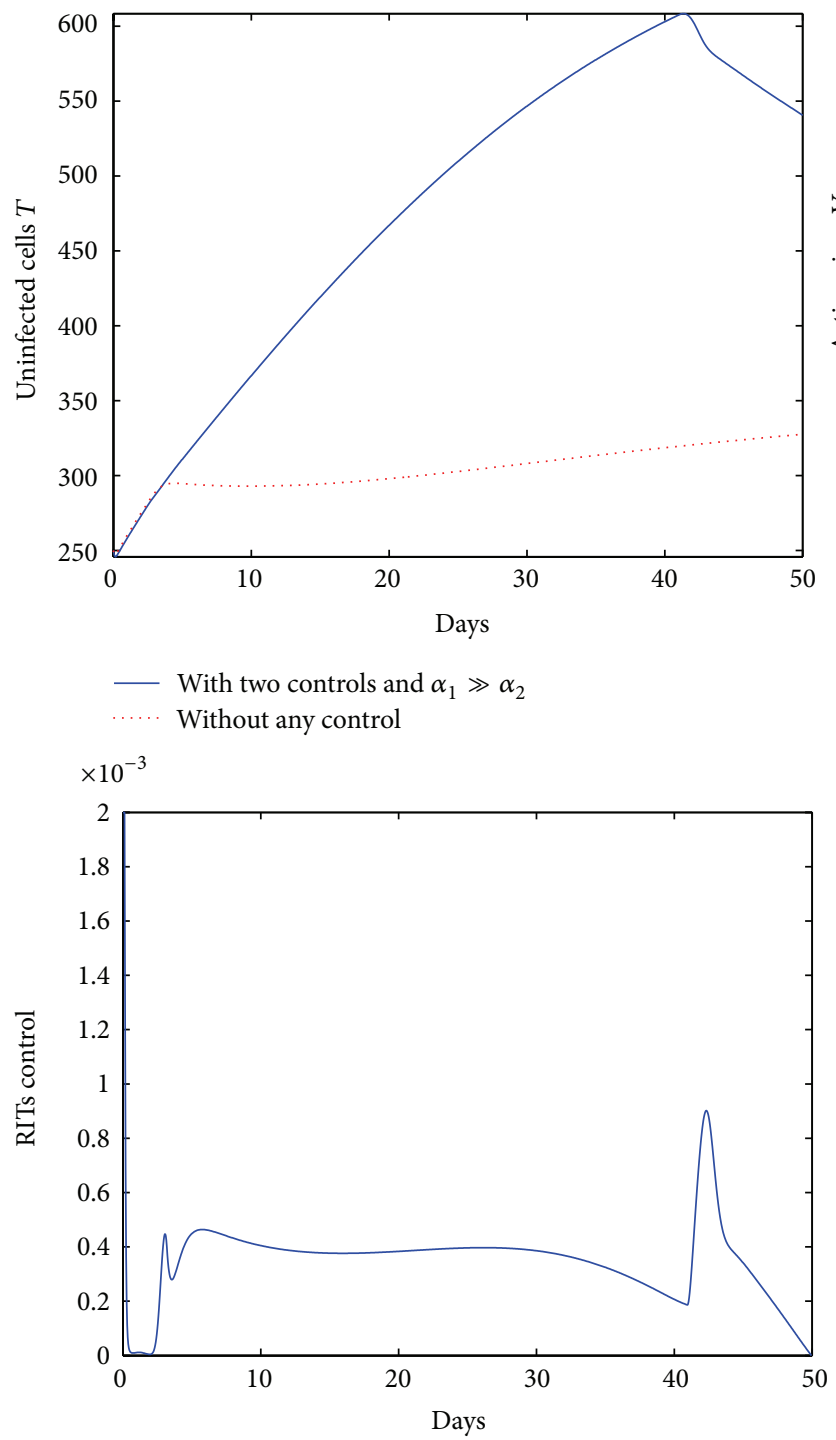
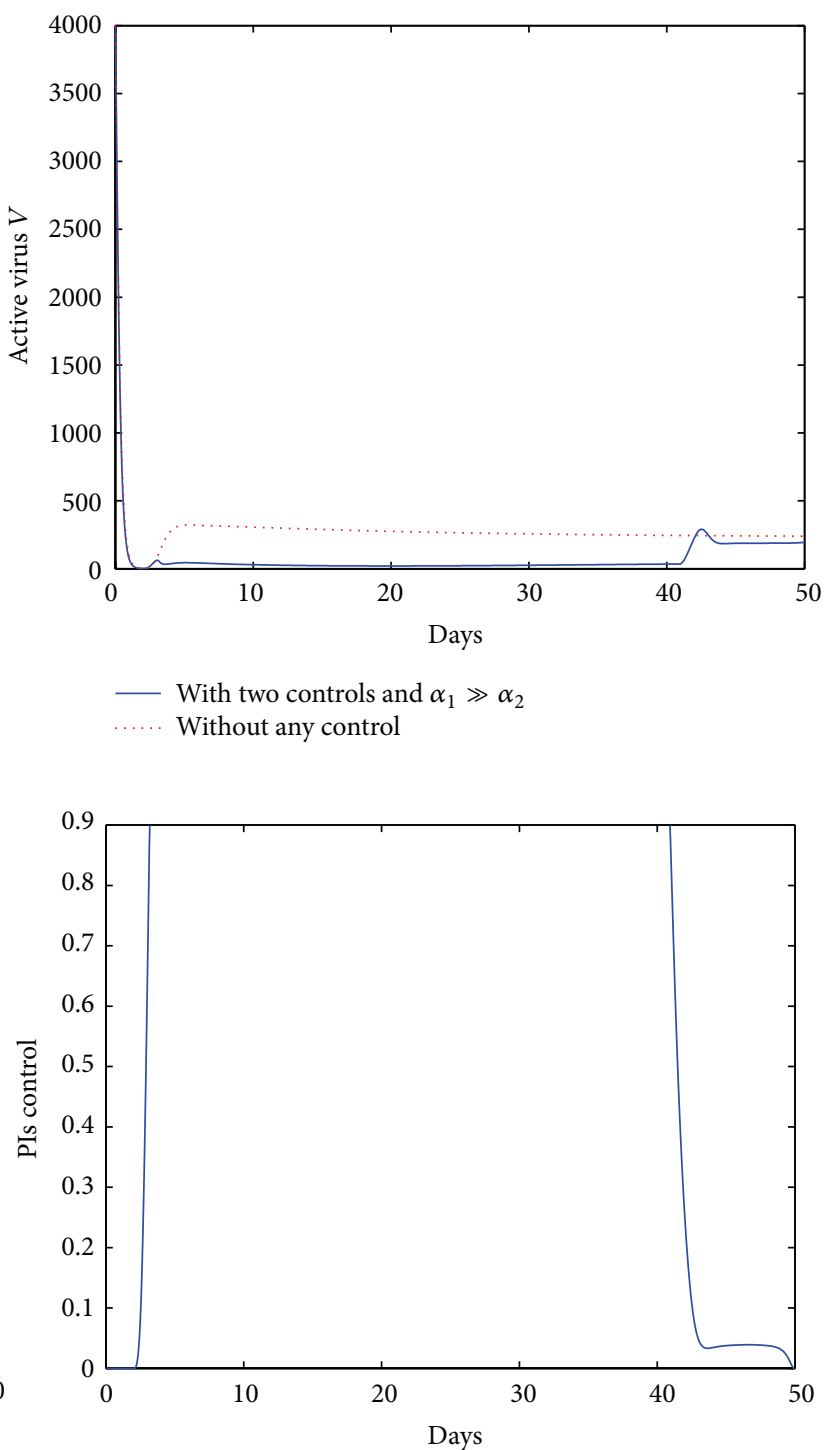

FIgURE 5: Optimal solutions using RTIs and PIs with $\alpha_{1} \gg \alpha_{2}$ for a 50-day treatment.

projection method to solve the optimal control problem numerically for an intuitive solution. The ODE is discrete with Euler discrete format and the costate equations of the recurrence equations produced by discretion are employed to calculate the gradient. The dynamic systems response is exactly computed with adjusted control history from one iteration to the next to increase objective function at each step. The iterations continue until convergence is achieved. The convergence criterion is the norm of the gradient projection on feasible control field. The convergence rate of this method is slow, but it is convergent in the problem of this paper.

Linked to the work in [4], we set the initial conditions $T(0)=250, L(0)=100, I(0)=200, V(0)=4000, E(0)=$ $100, B(0)=50 \mathrm{~mm}^{-3}$, weight factors $\left(\alpha_{1}, \alpha_{2}\right)$ are the combinations of 125000 and 125 , and the drug doses are up bounded by $b_{1}=0.002$ and $b_{2}=0.9$, respectively, a limitation is for the purpose of exploring optimal chemotherapy treatment to avoid an excessive use of drugs as indeed when these drugs are administered in high dose they are toxic to the human body and cause damage. We choose a 50-day treatment period in keeping with what is in [4] on treatment of HIV/AIDS. In the following sections, we will only draw the paths of uninfected $\mathrm{CD} 4^{+} \mathrm{T}$-cells $T$ and virus particles $V$, because people may be more interested in the number of uninfected $\mathrm{CD} 4^{+} \mathrm{T}$-cells and virus particles rather than other cells in clinical practice. Also, we will plot the path relative to $T$ or $V$ without any control at each drawing for comparing the treatment effect before and after the chemotherapy is introduced. This paper will illustrate nine cases for different combinations of the weight factors pairs $\left(\alpha_{1}, \alpha_{2}\right)$ combining with two kinds of upperbounds $b_{1}$ for a 50 -day treatment schedule. These strategies include:

(i) Strategy I: only use of PIs with sufficiently small $\alpha_{2}$;

(ii) Strategy II: only use of PIs with sufficiently large $\alpha_{2}$; 

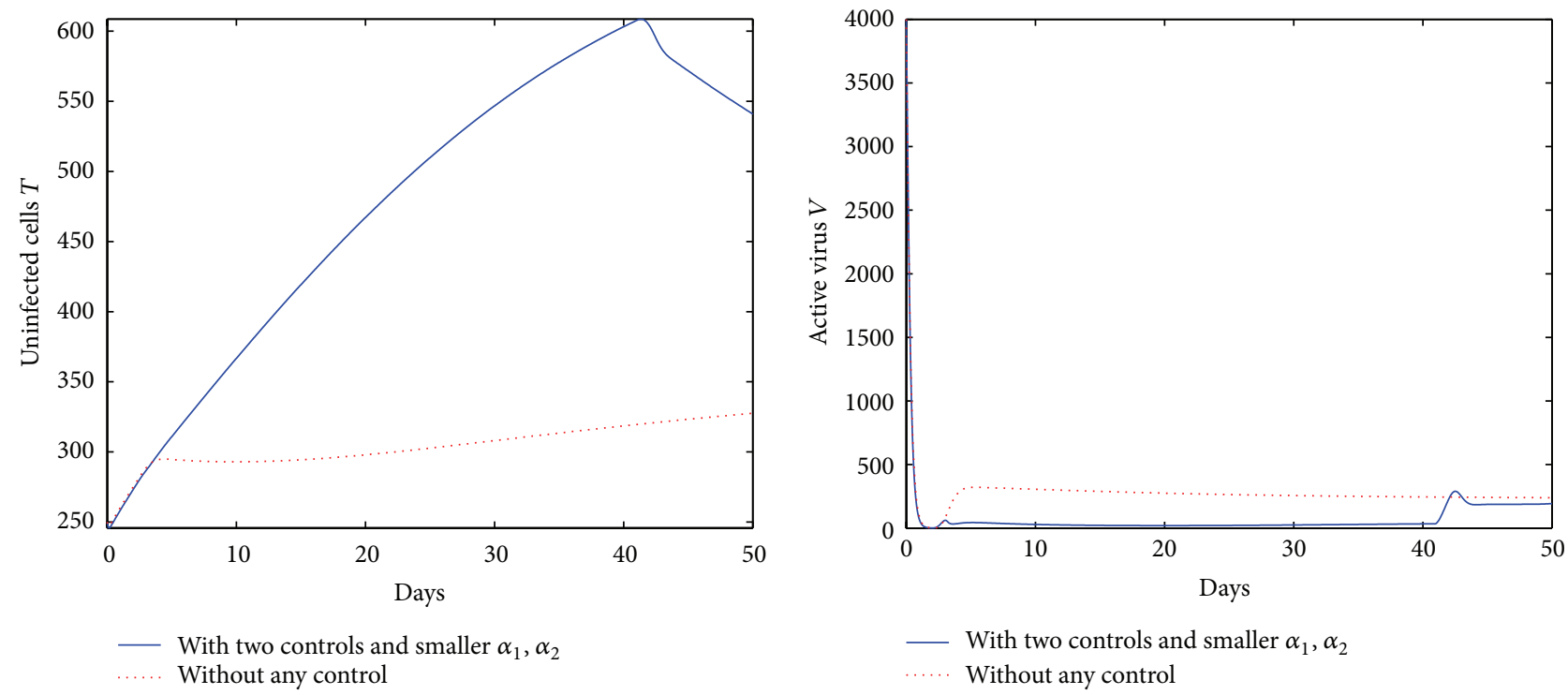

With two controls and smaller $\alpha_{1}, \alpha_{2}$

Without any control
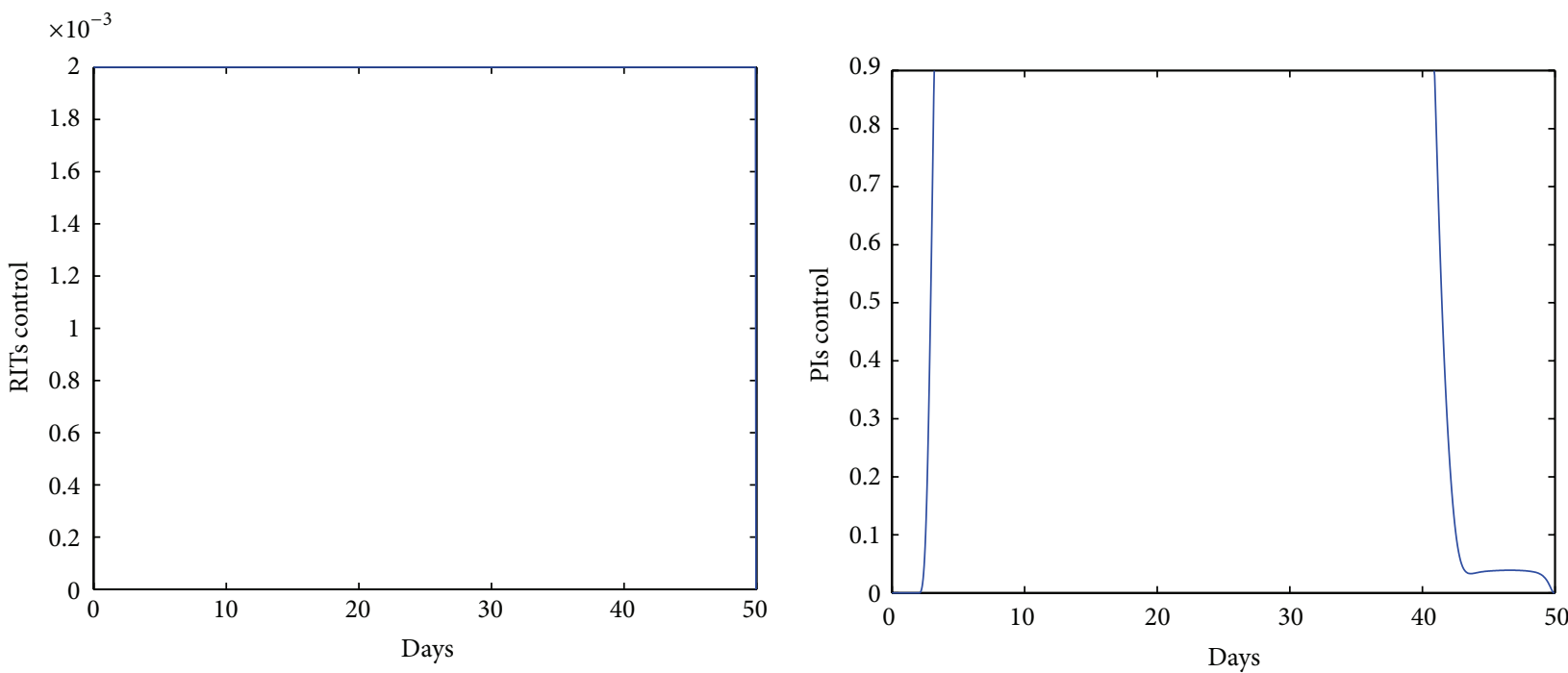

FIgURE 6: Optimal solutions using RTIs and PIs with sufficiently small $\alpha_{1} \approx \alpha_{2}$ for a 50-day treatment.

(iii) Strategy III: only use of RTIs with sufficiently large $\alpha_{1}$ and smaller $b_{1}$;

(iv) Strategy IV: only use of RTIs with sufficiently small $\alpha_{1}$ and smaller $b_{1}$;

(v) Strategy V: use both RTIs and PIs with $\alpha_{1} \gg \alpha_{2}$ and smaller $b_{1}$;

(vi) Strategy VI: use both RTIs and PIs with sufficiently small $\alpha_{1} \approx \alpha_{2}$ and smaller $b_{1}$;

(vii) Strategy VII: use both RTIs and PIs with $\alpha_{1} \ll \alpha_{2}$ and smaller $b_{1}$;

(viii) Strategy VIII: use both RTIs and PIs with sufficiently large $\alpha_{1} \approx \alpha_{2}$ and smaller $b_{1}$;

(ix) Strategy IX: use both RTIs and PIs with sufficiently small $\alpha_{1} \approx \alpha_{2}$ and larger $b_{1}$.
Remark 4. The extra Strategy IX is considered to compare the different treatment effects for the two-drug strategies with similar weight factors, but with different upper-bounds for the control $u_{1}$.

Let $u_{1} \equiv 0$ and let $\alpha_{2}=125$, then we can get the optimal treatment policy with single PIs, that is, Strategy I. Figure 1 is plotted by using $\alpha_{1}=0, \alpha_{2}=125, b_{1}=0$, and $b_{2}=0.9$ and keeping the rest of the parameters unchanged. The number of uninfected $\mathrm{CD} 4^{+} \mathrm{T}$-cells $T$ in Figure 1 is obviously higher than one without any control, while the number of virus particles $V$ is evidently lower than one without any control about 4 days after beginning therapy, which implies that the treatment only using PIs with sufficiently small weight coefficient can effectively improve the patients' condition although the treatment effect begins to reduce from the 41th day. The optimal drug administration schedule of PIs is with 

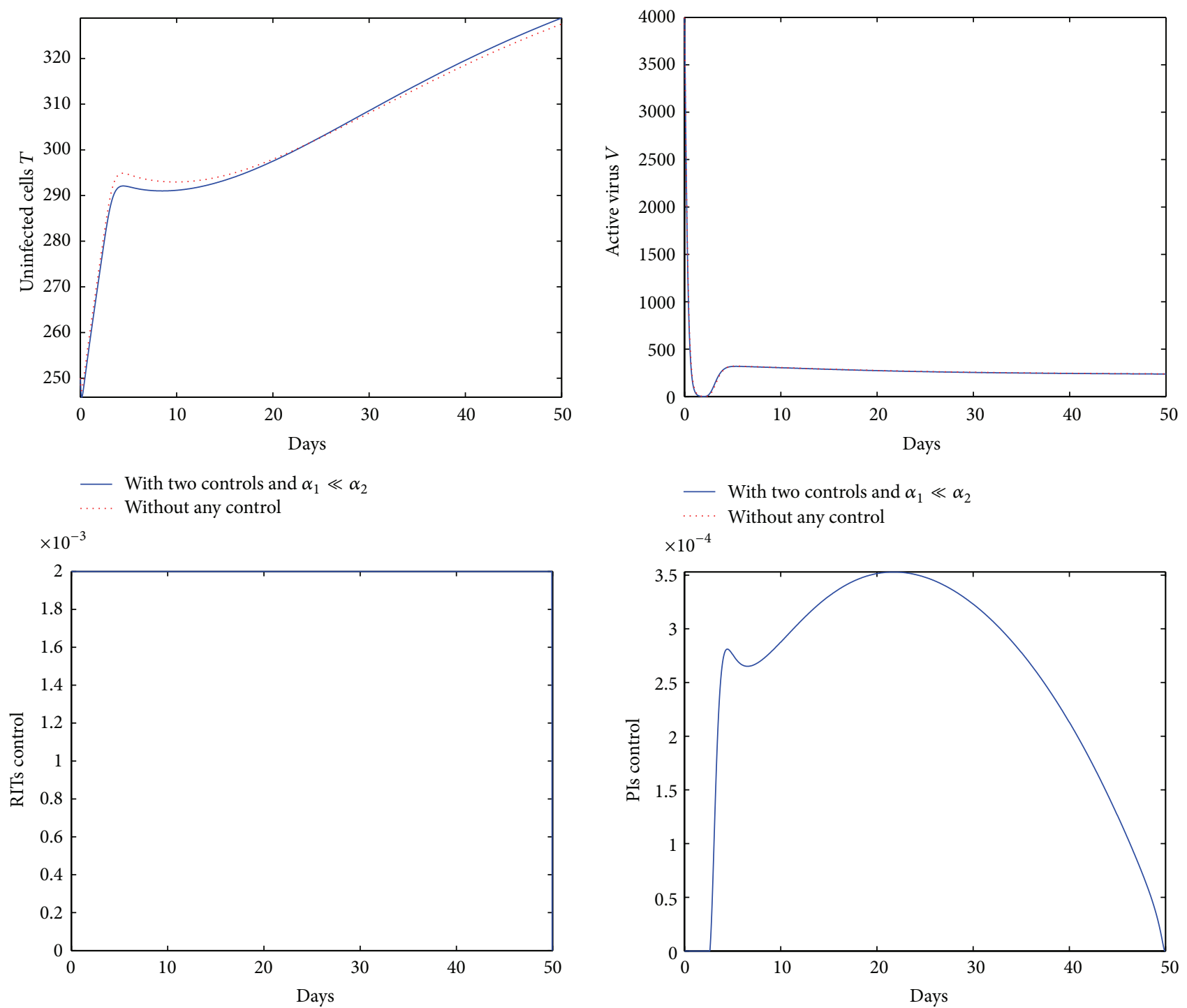

FIgURE 7: Optimal solutions using RTIs and PIs with $\alpha_{1} \ll \alpha_{2}$ for a 50-day treatment.

maximal effort for a longer period of about 38 days (4th-41th day), but with almost no treatment in the other times including initial stages and final stages.

Figure 2 is plotted using $\alpha_{1}=0, \alpha_{2}=125000, b_{1}=0$, and $b_{2}=0.9$, and keeping the rest of parameters unchanged under the assumption of $u_{1} \equiv 0$. Figure 2 represents the treatment policy only using PIs with sufficiently large weight coefficient, that is, Strategy II. The optimal schedule recommends that the dose of PIs is extremely low (less than 0.00035). But, the dynamics of uninfected CD $4^{+}$T-cells $T$ and active virus $V$ in Figure 2 hints no improvement for treatment. In other words, Strategy II is not useful for the therapy of HIV/AIDS.

Figure 3 is plotted by using $\alpha_{1}=125000, \alpha_{2}=0$, $b_{1}=0.002$, and $b_{2}=0$, and keeping the rest of parameters unchanged under the assumption of $u_{2} \equiv 0$. Figure 3 represents the treatment policy only using RTIs with sufficiently large weight coefficient, that is, Strategy III. The optimal schedule recommends that the dose of RTIs is decreasing.
But, the dynamics of uninfected $\mathrm{CD} 4^{+} \mathrm{T}$-cells $T$ and active virus $V$ in Figure 3 means no improvement for treatment.

Figure 4 is plotted by using $\alpha_{1}=125, \alpha_{2}=0, b_{1}=0.002$, and $b_{2}=0$, and keeping the rest of parameters unchanged under the assumption of $u_{2} \equiv 0$. Figure 4 represents the treatment policy only using RTIs with sufficiently small weight coefficient, that is, Strategy IV. The dynamics of uninfected $\mathrm{CD} 4^{+}$T-cells $T$ and active virus $V$ in Figure 4 implies no improvement for treatment, though the maximal effort for control is needed.

Figure 5 is plotted by using $\alpha_{1} \gg \alpha_{2}$ (for instance, $\alpha_{1}=$ 125000 and $\left.\alpha_{2}=125\right), b_{1}=0.002$, and $b_{2}=0.9$, and keeping the rest of the parameters unchanged. Figure 5 represents Strategy $\mathrm{V}$ and shows that the number of uninfected $\mathrm{CD} 4^{+} \mathrm{T}-$ cells $T$ is obviously added, while the number of virus particles $V$ is evidently reduced about 4 days after beginning therapy. The optimal schedule recommends that the dose of RTIs is extremely low (majority less than 0.0005 ) and in the opposite 

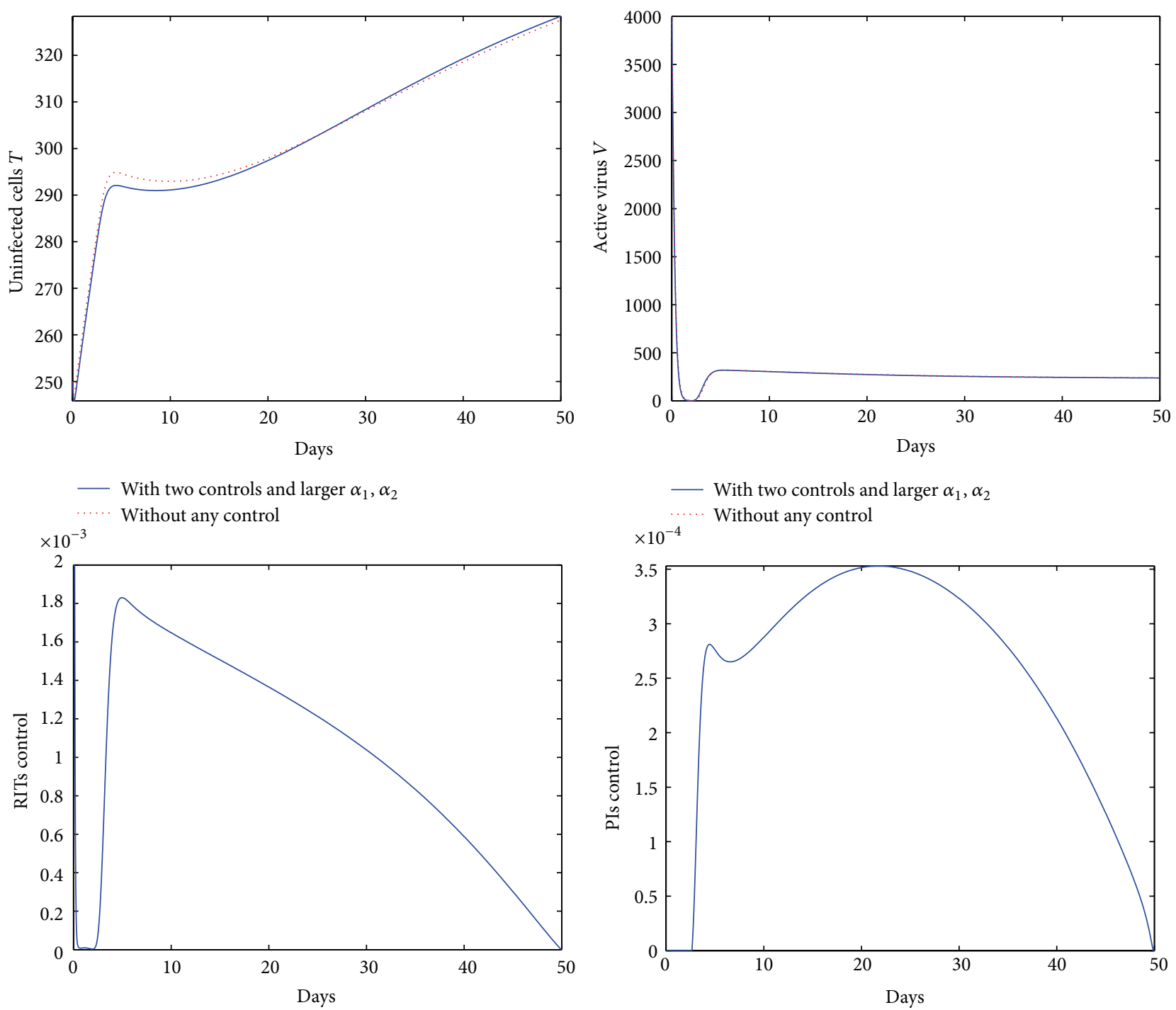

FIGURE 8: Optimal solutions using RTIs and PIs with sufficiently large $\alpha_{1} \approx \alpha_{2}$ for a 50-day treatment.

PIs is used with maximal effort during its valid term (4th41th day).

Figure 6 is plotted using sufficiently small $\alpha_{1} \approx \alpha_{2}$ (for instance, $\alpha_{1}=125$ and $\alpha_{2}=125$ ), $b_{1}=0.002$, and $b_{2}=0.9$, and keeping the rest of the parameters unchanged. Figure 6 represents Strategy VI and gives the similar results as Strategy $\mathrm{V}$. But the optimal schedule recommends that the dose of RTIs is used with maximal effort during almost whole treatment period and PIs is used with maximal effort during its valid treatment term (4th-41th day).

Figure 7 is plotted using $\alpha_{1} \ll \alpha_{2}$ (for instance, $\alpha_{1}=125$ and $\left.\alpha_{2}=125000\right), b_{1}=0.002$, and $b_{2}=0.9$, and keeping the rest of the parameters unchanged. Figure 7 represents Strategy VII and shows that the number of uninfected $\mathrm{CD} 4^{+} \mathrm{T}$-cells $T$ is not yet added, while the number of virus particles $V$ is not yet reduced though two-drug treatment has been implemented during whole therapy period. This means that the Strategy VII is not at all helping the therapy of HIV/AIDS.
Figure 8 is plotted using sufficiently large $\alpha_{1} \approx \alpha_{2}$ (for instance, $\alpha_{1}=125000$ and $\left.\alpha_{2}=125000\right), b_{1}=0.002$, and $b_{2}=0.9$, and keeping the rest of the parameters unchanged. Figure 8 represents Strategy VIII and gives a similar ineffective-treatment result as Strategy VII, although two-drug treatment is implemented.

Remark 5. From Figures 1-8, we find that no strategies except Strategy I, Strategy V, and Strategy VI are very helpful for the therapy of HIV/AIDS, which means that all of the single-drug treatment strategies with RTIs are ineffective, or at least are not very effective. These theoretical evidences also show the fact that using a single RTIs is not an effective clinical strategy for therapy of HIV/AIDS.

Remark 6. We also find that the treatment policies with sufficient large weight coefficients are all useless, or at least are not particularly helpful, which implies that high costs may have critical implications for the treatment of HIV. 

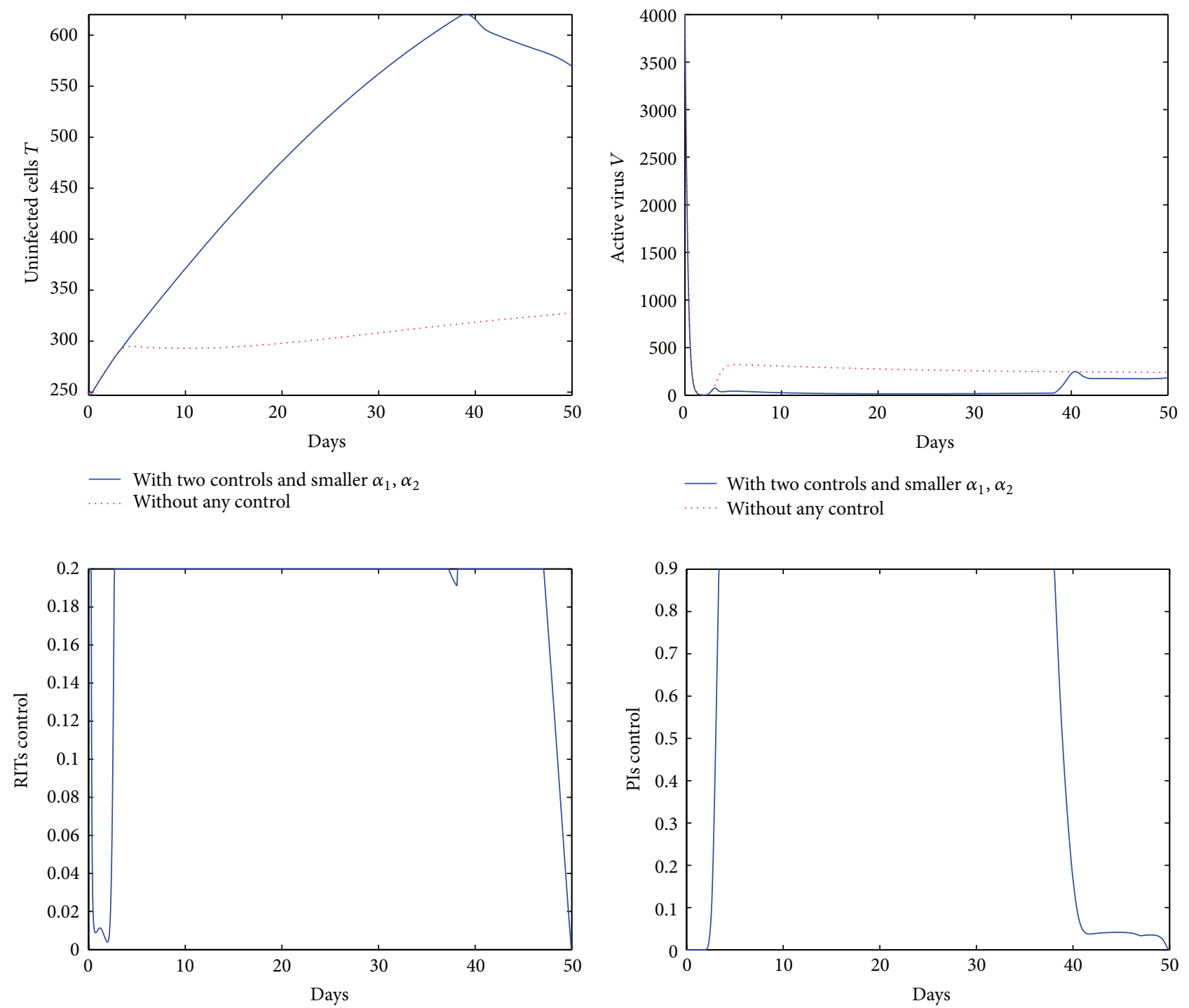

FIGURE 9: Optimal solutions using RTIs and PIs with sufficiently small $\alpha_{1} \approx \alpha_{2}$ and bigger upper-bound $b_{1}$ for a 50-day treatment.

Moreover, among the strategies with effective treatment, the treatment effect using Strategy V and Strategy VI (with twodrug control) seems similar as the one using Strategy I (with single-drug control (PIs)). Is it really true? When the upper-bound $b_{1}$ for control $u_{1}$ in the strategies with twodrug treatment is added (a hundredfold, for instance), the Strategy VI can get better treatment effect than the Strategy I, which implies that two-drug control strategy with small weight factors, when RTIs are improved but PIs unchanged, is more effective than single-drug control strategy with PIs. Figure 9 (Strategy IX) illustrates this case. But, whatever the upper-bound $b_{1}$ is, the treatment effect using Strategy $\mathrm{V}$ (with large weight factor for RTIs) is almost consistent with the one using Strategy I, which shows, again, that large weight factor (i.e., high cost) may counteract treatment effect.
Remark 7. Comparing, respectively, the maximal value of $T$ (608) and the minimal value of $V(40)$ in Figure 6 with the maximal value of $T$ (620) and the minimal value of $V$ (21) in Figure 9, we find that the treatment effect for the strategy using both RTIs and PIs with sufficiently small weight factors has not gotten remarkably improved even after the upperbound $b_{1}$ for the control $u_{1}$ is increased a hundredfold. This implies that only increasing dosage of RTIs for the effective two-drug control strategies cannot markedly improve the patient's health condition.

Remark 8. Overall, Strategy V, Strategy VI, and Strategy IX show that combined treatment such as above two-drug therapy is more effective than single-drug cure. This fact may help to explain why the HAARP is still the most effective method for therapy of HIV/AIDS till now. 


\section{Conclusion and Discussion}

In this research, a deterministic HIV model including immune response, neutralizing antibodies, and multidrug effects is improved. Optimization theories are used to derive optimal control solution and to design improved clinical treatments. The existence for the optimal control problem is proved, the optimality system is derived, and a gradient projection method is applied to numerically simulate different therapy effects. On the basis of combinations of weight factors and upper-bounds for controls, we establish some of much interesting or even strange treatment strategies including four types of single-drug controls and five types of twodrug controls, where a large weight coefficient means a high cost of the corresponding drug. Among the nine strategies, no strategy except the Strategy I, Strategy V, Strategy VI, and Strategy IX is helpful for the therapy of HIV/AIDS, which means that the single-drug treatment with RTIs is ineffective for treating HIV/AIDS, or at least is not very effective. These theoretical evidences also show the fact that using a single RTIs is not an effective clinical strategy for therapy of HIV/AIDS. We also find that the treatment policies with higher cost are not useful, or at least are not particularly helpful. Among those strategies with effective treatment, the policy using two-drug control with lower cost should be the best clinical one because it can more efficiently increase the number of uninfected $\mathrm{CD} 4^{+} \mathrm{T}$-cells and decrease the number of virus particles. And, overall, combined treatment is more effective than single-drug cure. This fact may help to explain why the HAARP is always regarded as the most effective method for therapy of HIV/AIDS.

But, why can the larger weight factor counteract the use of drug? Is it possible that, as indicated by Figure 2 and Figure 8 , the higher cost results in the lower dosage of drug? For the effective two-drug treatment policies, why has the treatment effect not gotten markedly improved even after the dosage of RTIs is increased manyfold? Further researches need to be done in the future.

\section{Conflict of Interests}

The authors declare that there is no conflict of interests regarding the publication of this paper.

\section{Acknowledgments}

This research was supported by the Natural Science Foundation of China (11271371) (71272209), and the Humanity and Social Science Foundation of Ministry of Education of China (12YJA630170), and the Natural Science Foundation of Hunan Province (12JJ3081). Thank are due to the reviewer's comments for this paper.

\section{References}

[1] B. M. Adams, H. T. Banks, H. Kwon, and H. T. Tran, "Dynamic multidrug therapies for HIV: optimal and STI control approaches," Mathematical Biosciences and Engineering, vol. 1, no. 2, pp. 223-241, 2004.
[2] R. Zurakowski and A. R. Teel, "A model predictive control based scheduling method for HIV therapy," Journal of Theoretical Biology, vol. 238, no. 2, pp. 368-382, 2006.

[3] J. Karrakchou, M. Rachik, and S. Gourari, "Optimal control and infectiology: application to an HIV/AIDS model," Applied Mathematics and Computation, vol. 177, no. 2, pp. 807-818, 2006.

[4] W. Garira, S. D. Musekwa, and T. Shiri, "Optimal control of combined therapy in a single strain HIV-1 model," Electronic Journal of Differential Equations, vol. 2005, no. 52, pp. 1-22, 2005.

[5] D. Hull, Optimal Control Theory for Applications, Springer, New York, NY, USA, 2003.

[6] M. I. Kamien and N. L. Schwartz, Dynamic Optimization: The Calculus of Variations and Optimal Control in Economics and Management, Advanced Textbooks in Economics, NorthHolland Press, Amsterdam, Netherlands, 1991.

[7] L. S. Pontryagin, Mathematical Theory of Optimal Processes, CRC Press, Boca Raton, Fla, USA, 1987.

[8] Y. Lin and P. Zhong, "Impact, mechanissm and laboratory detection of neu tralizing antibody in HIV-1," Chinese Journal of AIDS \& STD, vol. 14, no. 6, pp. 634-636, 2008 (Chinese).

[9] J. S. McLellan, M. Pancera, C. Carrico et al., "Structure of HIV-1 gp120 V1/V2 domain with broadly neutralizing antibody PG9," Nature, vol. 480, no. 7377, pp. 336-343, 2011.

[10] C. Ren, Y. Li, and H. Ling, "Neutralizing anti bodies responses during natural HIV-1 infection," International Journal of Immunology, vol. 3, no. 35, pp. 176-179, 2012 (Chinese).

[11] Y. Zhou, Y. Liang, and J. Wu, "An optimal strategy for HIV multitherapy," Journal of Computational and Applied Mathematics, vol. 263, pp. 326-337, 2014.

[12] J. M. Orellana, "Optimal control for HIV multitherapy enhancement," Comptes Rendus Mathematique, vol. 348, pp. 1179-1183, 2011.

[13] H. Wu, H. Zhu, H. Miao, and A. S. Perelson, "Parameter identifiability and estimation of HIV/AIDS dynamic models," Bulletin of Mathematical Biology, vol. 70, no. 3, pp. 785-799, 2008.

[14] M. A. Stafford, L. Corey, Y. Cao, E. S. Daar, D. D. Ho, and A. S. Perelson, "Modeling plasma virus concentration during primary HIV infection," Journal of Theoretical Biology, vol. 203, no. 3, pp. 285-301, 2000.

[15] W. H. Fleming and R. W. Rishel, Deterministic and Stochastic Optimal Control, Springer, New York, NY, USA, 1975.

[16] D. L. Lukes, "Differential equations: classical to controlled," in Mathematics in Science and Engineering, Academic Press, New York, NY, USA, 1982.

[17] A. S. Perelson, D. E. Kirschner, and R. D. Boer, "Dynamics of HIV infection of $\mathrm{CD} 4^{+} \mathrm{T}$ cells," Mathematical Biosciences, vol. 114, no. 1, pp. 81-125, 1993. 


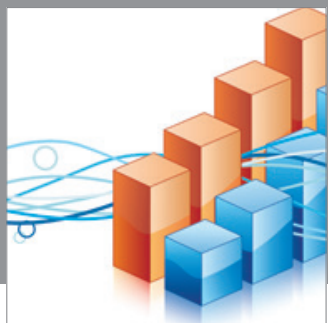

Advances in

Operations Research

mansans

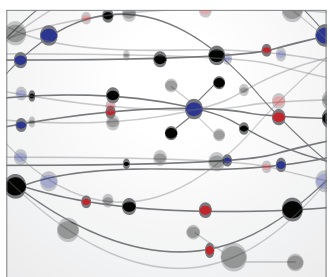

The Scientific World Journal
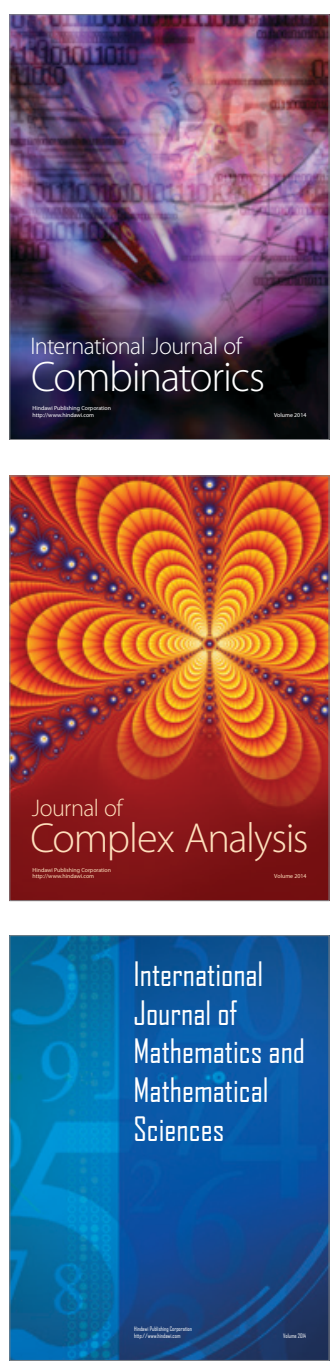
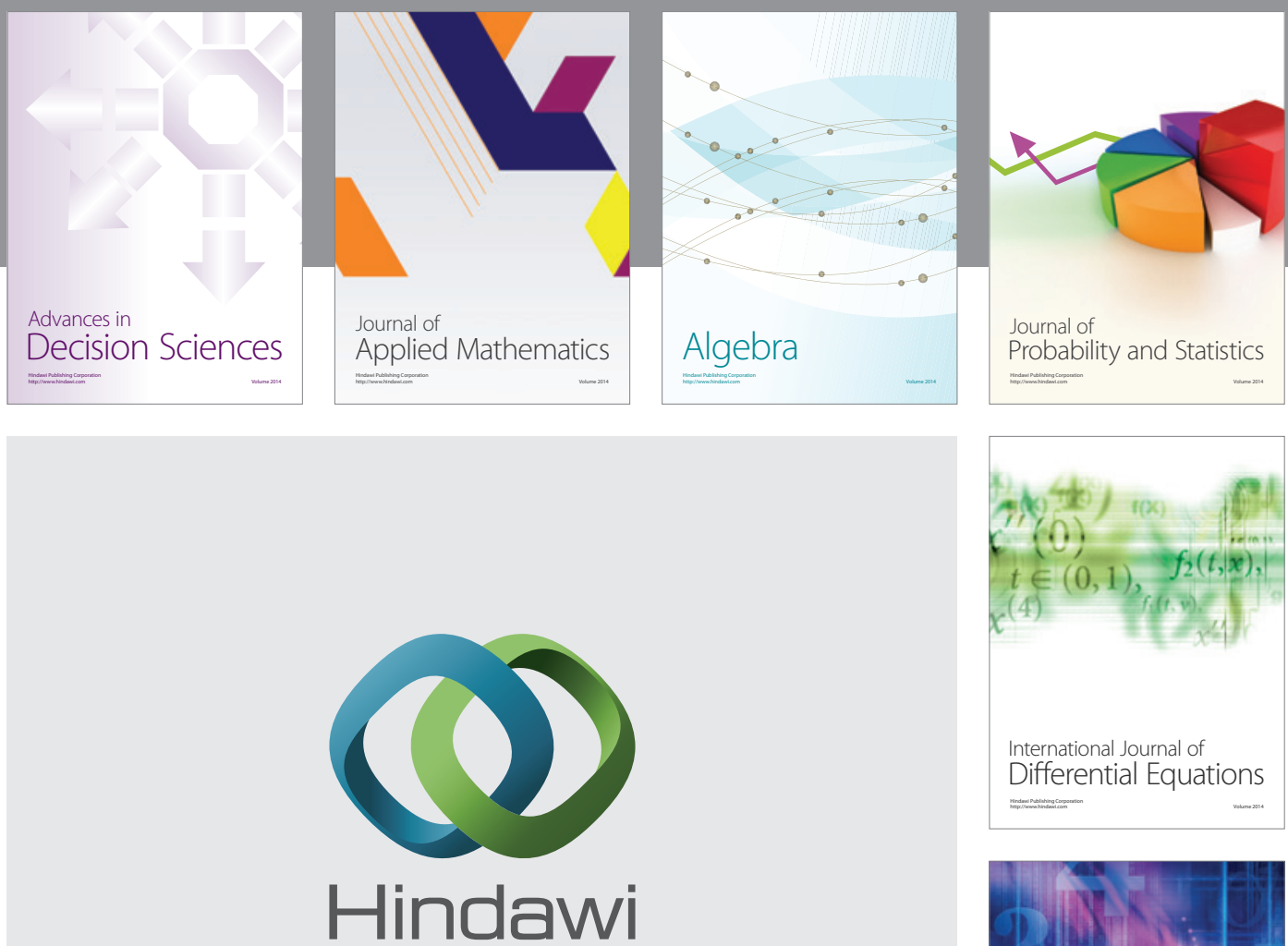

Submit your manuscripts at http://www.hindawi.com
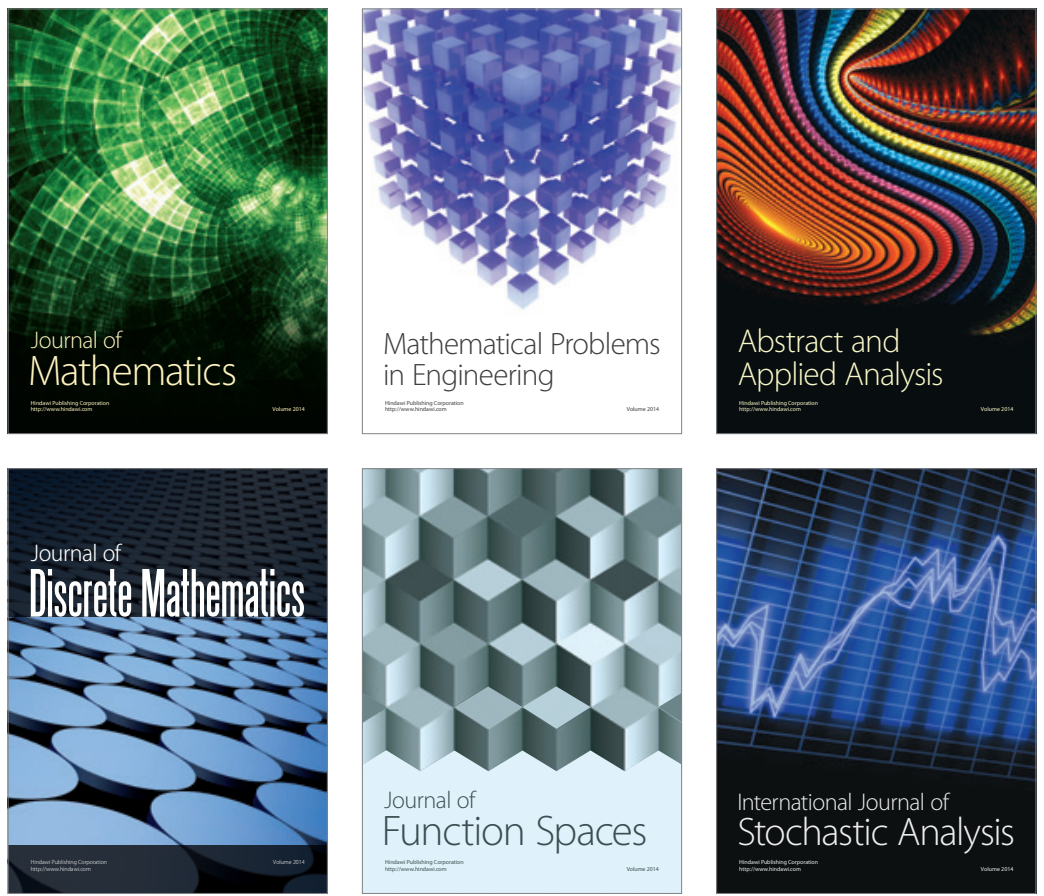

Journal of

Function Spaces

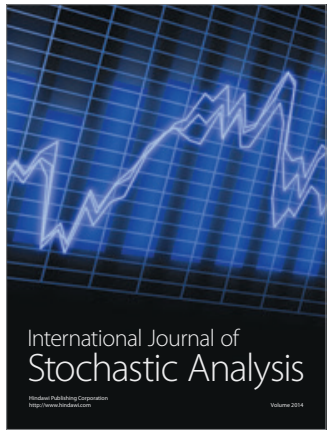

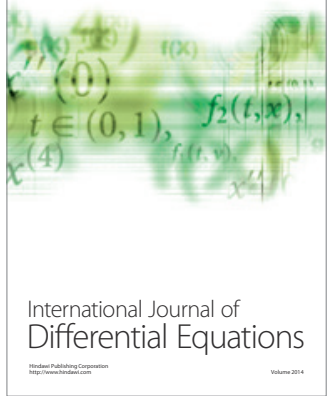
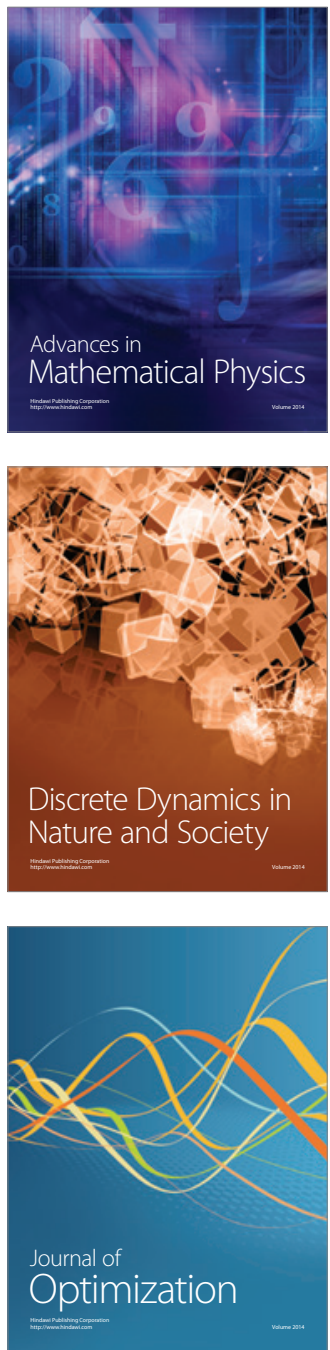